\title{
Multiple Baseline Radar Interferometry Applied to Coastal Land Cover Classification and Change Analyses
}

\author{
Elijah Ramsey, III ${ }^{1}$ \\ USGS National Wetlands Research Center, 700 Cajundome Blvd., Lafayette, \\ Louisiana 70506
}

\section{Zhong Lu}

Science Applications International Corporation (SAIC), USGS Center for Earth Resources Observation and Science (EROS), Sioux Falls, South

Dakota 57198

\section{Amina Rangoonwala}

IAP World Services, Inc., Lafayette, Louisiana 70506

\section{Russell Rykhus}

Science Applications International Corporation (SAIC), USGS Center for Earth Resources Observation and Science (EROS), Sioux Falls, South Dakota 57198

\begin{abstract}
ERS-1 and ERS-2 SAR data were collected in tandem over a four-month period and used to generate interferometric coherence, phase, and intensity products that we compared to a classified land cover coastal map of Big Bend, Florida. Forests displayed the highest intensity, and marshes the lowest. The intensity for fresh marsh and forests progressively shifted while saline marsh intensity variance distribution changed with the season. Intensity variability suggested instability between temporal comparisons. Forests, especially hardwoods, displayed lower coherences and marshes higher. Only marshes retained coherence after 70 days. Coherence was more responsive to land cover class than intensity and provided discrimination in winter. Phase distributions helped reveal variation in vegetation structure, identify broad land cover classes and unique within-class variations, and estimate water-level changes.
\end{abstract}

\section{INTRODUCTION}

Coastal regions are some of the world's most diverse, dynamic, and complex natural environments. From a regional monitoring perspective, the dynamic nature of coastal areas requires a high observation frequency to capture abnormal events and

\footnotetext{
${ }^{1}$ Email: elijah_ramsey@usgs.gov
} 
underlying processes. Numerous approaches have been developed to map complex coastal regions using remote sensor data. At a minimum, an applicable remote sensing system should provide data daily to weekly and at $1.0-30.0 \mathrm{~m}$ spatial resolutions. While airborne systems offer the best method to fulfill these data constraints, they are costly and availability is limited. In addition, weather conditions often constrain airborne collections and, as with optical satellite systems, degrade data quality.

Numerious studies have shown that passive optical systems with a moderate spatial resolution (e.g., Landsat Thematic Mapper) can adequately address many regional resource management issues, including land cover changes, and biophysical processes (Jensen et al., 1987; Klemas et al., 1993; Lunetta et al., 1998; Ramsey et al., 2001b). Although moderate-resolution mapping cannot yet quantify the amount and types of change within highly heterogeneous coastal landscapes (Ramsey and Laine, 1997), multiple source integration and sub-pixel extraction are improving the spatial detail (Ramsey et al., 1998; Ramsey et al., 2005). Temporal constraints are more problematic. Time series analyses and sensor combinations have improved the temporal content of regional mapping and of specific events or broad changes (e.g., flooding, storms, drought) (Ramsey et al., 2001a); however, the overriding constraint for optical systems is clouds, especially in subtropical regions and during severe weather events (Ramsey, 1995).

The failure of optical remote sensing systems to provide a consistent data source is a critical issue in coastal resource management (Ramsey and Laine, 1997). Operational management and proper resource evaluation require that data be collected consistently, not opportunistically. Even when reliance on time-constrained collections is minimized, the potential to maximize extractable information or capture timedependent features is limited using optical systems (Moghaddam and McDonald, 2003).

Satellite microwave data offer a good alternative data source when timely collection is the dominant concern (Lyon and McCarthy, 1981; Kasischke and BourgeauChavez, 1997; Ramsey, 1998). Active radar (radio detection and ranging) imaging systems operating within the microwave spectrum $(\sim 1.0-150 \mathrm{~cm})$ can collect day and night and in nearly all weather conditions. As the satellite moves along its orbital path (azimuth direction), the radar antenna transmits a microwave pulse at an angle orthogonal to its flight direction (range direction) and then records the "backscatter" (i.e., returned signal intensity). Synthetic Aperture Radar (SAR) transmitted and received pulses are fully coherent, allowing the precise measurement of time-delay (slant range distance) and phase difference with respect to the reference phase (radar oscillator) (Baran, 2004). Operational SAR spatial resolutions (20-30 m) equal or exceed current regional optical satellite systems when scene coverage extends over $100 \mathrm{~km}$.

\section{Land Cover Mapping with SAR Intensity Data}

Radar backscatter intensity from a target is a function of the frequency or wavelength, imaging geometry, topography, surface roughness, and dielectric constant (Waring et al., 1995; Ramsey, 1998; Baran, 2004; Ramsey, 2005). Transformed to decibel units $(\mathrm{dB})$, the more positive the intensity value, the greater the amount of energy focused back toward the receiver. With respect to wavelength, intensity 
increases with increased surface roughness and with the dielectric constant of surface materials. The dielectric constant indicates the response of a material to the presence of an electric field (the radar pulse). In terrestrial mapping, the material water content dominates the dielectric constant. In essence, outside of geometric and topographic influences, the water held in the soil horizons and tree or grass canopies dominates the radar interaction and three-dimensional distribution of the intensity.

SAR systems encompass a variety of operating wavelengths, polarizations, incident angles, and spatial resolutions. The selective combination of sensor parameters uniquely identifies each SAR system and largely characterizes the type and range of land cover information available or extractable from each system (Elachi, 1988; Ulaby and Dobson, 1989; Dobson et al., 1995; Lewis et al., 1998; Ramsey, 1998; Jensen, 2000). Most SAR systems operate with a limited set of parameters hindering the widespread application of these systems in resource management. This constraint is akin to an optical sensor system restricted to a single wavelength at a fixed spatial resolution and reoccupation frequency. A SAR analog of the spectral extension of optical systems would combine multiple frequencies, multiple polarizations, and multiple incidence angles. Outside the Shuttle Imaging Radar (SIR) missions, and the Environmental Satellite (ENVISAT) and Advanced Land Observing Satellite (ALOS), most satellite SARs are single frequency, single polarization, with a nominally fixed incident angle and reoccupation frequency. Although multiple frequency and polarization SAR systems are planned for launch, their set of sensor parameters will still be limited.

\section{Interferometic Land Cover Mapping}

While the terrestrial mapping capabilities of polarimetric SAR (multiple polarimetric returns) have been extensively studied and quantified, the capabilities of interferometric data with regard to terrestrial mapping have not been adequately addressed (Hajnsek et al., 2003). SAR interferometry is the collection and processing of two SAR images of the same scene captured from two spatially separate positions (Armour et al., 1998). The separation or baseline is the relative difference in position of two receiving antennas on a single platform (single-pass) or from one or two platforms (repeat-pass). Repeat-pass interferometry uses two parallel or nearly parallel flight-path collections over the same area.

The interferogram is the complex cross-correlation of returns (electric fields) from the two ends of the baseline. The cross-correlation is used to estimate the degree to which the two SAR images are correlated. Normalized by the square roots of the two baseline intensities, transforms the correlation into coherence that varies between 0.0 and 1.0 (Treuhaft et al., 2002). After removal of baseline, geometric, and system dependencies, the coherence furnishes the confidence of the phase measurements (Hagberg et al., 1995; Wegmuller and Werner, 1997; Cloude and Papathanassiou, 1998; Hajnsek et al., 2003; Baran, 2004). The phase is an intrinsic component of the complex return at both ends of the baseline due to the two-way wave propagation (Hagberg et al., 1995).

The phase is controlled mainly by five effects: (1) differences in the satellite orbits in the two passes; (2) topography; (3) ground deformation; (4) atmospheric propagation delays; and (5) systematic and environmental noises. Satellite position 
and attitude are used to remove effects due to differences in the orbital passes and a digital elevation model is used to remove effects due to topographic variation (Massonnet and Feigl, 1998). The resultant phase then contains information related to (a) surface deformation (e.g., land subsidence or water level change), (b) surface vegetation structure that is relative to the DEM, and (c) noise.

Following the removal of the effect of bare-earth topography (DEM) from the original interferogram, the phase value can be approximated as:

$$
\delta \phi=-\frac{4 \pi}{\lambda} \frac{B_{\perp}}{H \tan \theta} \delta h-\frac{4 \pi}{\lambda} \delta d
$$

where $H$ is the satellite altitude above the reference earth surface, $B_{\perp}$ is the perpendicular component of the baseline with respect to the incidence angle $\theta$; $\lambda$ is the wavelength of SAR, $\delta h$ is surface vegetation height, and $\delta d$ is the surface deformation or change in water level along the radar's look direction. In the ERS SAR images used in this study, $H$ is approximately $787.6 \mathrm{~km}, \theta$ is approximately $21.5^{\circ}, \lambda$ is $5.66 \mathrm{~cm}$, and $B_{\perp}$ should be less than $1100 \mathrm{~m}$ for a coherent interferogram. Equation 1 can be approximated as:

$$
\delta \phi \approx-\frac{1}{1400} B_{\perp} \delta h-\frac{4 \pi}{\lambda} \delta d
$$

From Equation 2, it is evident that the repeat-pass phase value can be more sensitive to changes in topography (i.e., $\delta d$ ) than to the topography itself (i.e., $\delta h$ ).

Four images make up the interferometric product: two intensity images, one phase image, and one coherence image. All images contain noise: primarily speckle, an artifact of the coherent SAR system, and thermal noise, represented by the signalto-noise ratio (SNR) of the system. The SNR magnitude concerns mainly low intensity regions (Cloude and Papathanassiou, 1998). The dominant noise source in most imaging radars is speckle, which arises from the constructive and destructive interference of two coherent waves.

Although adjacent pixels may contain nearly identical compositions and distribution of scatterers, a slightly different view geometry (or look) produces somewhat different returns that are portrayed over an image as a spatial pattern of speckle (Lee et al., 2003). While all products are affected, coherence and phase images are less affected because speckle is widely eliminated when the two images are combined to form the interferogram (Hagberg et al., 1995; Hajnsek et al., 2003). This reduction implies that phase and coherence products are more spatially consistent than intensity and, as a result, they can provide better discrimination and range of land cover parameters (Hajnsek et al., 2003).

Other factors also differentially affect the performance of Inferometric SAR (InSAR) systems and the quality of the products. Factors that increase phase uncertainty are commonly described as decorrelation factors, including atmospheric, geometric (mainly baseline), temporal, and volume decorrelations (Wegmuller and Werner, 1997). Atmospheric decorrelation results when tropospheric and ionospheric 
fluctuations alter the microwave velocity (Armour et al., 1998; Massonnet and Feigl, 1998). Baseline decorrelation (target rotation from different look angles) increases fading and speckle noise in the phase (Allen, 1995; Wegmuller and Werner, 1997; Cloude and Papathanassiou, 1998). Baseline decorrelation increases as the perpendicular baseline lengthens to critical point at which coherence is lost (Zebker and Villasenor, 1992; Lu and Freymueller, 1998). In ERS interferometric analyses, the baseline limit is about $1100 \mathrm{~m}$ over urban and nonvegetated areas; however, it can be appreciably lower over vegetation (Hoen and Zebker, 2000) reducing the comparability of coherence images generated from highly different baselines.

Temporal decorrelation is produced by any event that changes the physical orientation, composition, or scattering characteristics and scattering distribution of scatterers within a return volume (Allen, 1995; Hagberg et al., 1995; Papathanassiou and Cloude, 2003). In land cover mapping, these decorrelations are primarily caused by wind changing the leaf and twig orientations; moisture and rain changing the dielectric constant; flooding changing the dielectric properties and roughness of the canopy background; seasonal phenology; growth (Waring et al., 1995; Wegmuller and Werner, 1997; Liu et al., 2004); and mechanical changes such as cultivation and timber harvesting (Wegmuller and Werner, 1997). Inherent in the temporal dependencies are the stability of the scatterers, especially nearer the top-of-canopy (TOC) and the dependency with respect to the wavelength (Hagberg et al., 1995; Wegmuller and Werner, 1997; Papathanassiou and Cloude, 2003). Complete temporal decorrelation occurs when scatterer movements are higher than one-half the wavelength or 2.0-3.0 $\mathrm{cm}$ at $\mathrm{C}$ band (Hagberg et al., 1995; Liu et al., 2004).

Volume decorrelation can be viewed as a component of all previous decorrelation types, except possibly atmospheric decorrelation (Cloude and Papathanassiou, 1998). Volume scattering decreases coherence (Hagberg et al., 1995), and as a consequence, degrades the phase measurement. Volume scattering is produced by multiple scattering of the radar pulse that occurs within a distributed volume, such as forest and grass canopies, soil, and ice (Ramsey, 2005). The contribution of volume scattering is largely controlled by the proportion of signal that penetrates the surface and the twoway attenuation from the surface to the volume element. Relative to described SAR parameter dependences, penetration, and attenuation can broadly be expressed as functions of the surface roughness and volume extinction coefficient (largely a function of canopy density, structure, and moisture content). As the penetration depth decreases, the phase center as a vector combination modulated by the volume extinction coefficient shifts more toward the TOC (Hagberg et al., 1995; Treuhaft et al., 2002; Treuhaft et al., 2004).

Coherence and phase offer unique information not available in intensity products that may be useful for classifying land covers (Hagberg et al., 1995; Wegmuller and Werner, 1997). Even though beset by ambiguities and although the degradation of coherence is not well understood (Hagberg et al., 1995), decorrelation, as it influences coherence, may have a unique association with cover types. For instance, in the winter, sparse or bare fields and grasslands exhibited high coherence (Wegmuller and Werner, 1997), while forests exhibited lower coherence and phase height shift because of increased volume decorrelation (Hagberg et al., 1995). Also in the winter, deciduous forests exhibited a higher coherence than did coniferous forests (Wegmuller and Werner, 1997). Forest flooding boosts the double-bounce mechanism 
enhancing the return to the sensor and coherence promoting the detection of subcanopy flooding and water-level change (Ramsey, 1998). Whereas these spatial and temporal responses to land cover features can sometimes agree and sometimes differ with intensity data, these interferometric data augment intensity information and provide information not available with intensity data alone (Wegmuller and Werner, 1997).

\section{Study Objectives}

Operational resource mapping requires a spatially and temporally acceptable data source that is unhampered by weather or cloud conditions and can provide adequate information on coastal resource status and change. To take advantage of the full potential of imaging SAR and to increase the information content of past SAR collections, this paper discusses the inclusion of InSAR and its derivative information on phase, coherence, and amplitude. InSAR can provide a nearly unhampered data source; however, interferometric products constructed from a single image pair cannot provide unambiguous land cover information. To help alleviate this ambiguity, multiple wavelength, polarization, and baseline interferometric approaches were tested (Cloude and Papathanassiou, 1998; Reigber et al., 1999). We extended these studies to a coastal region that includes coastal and inland marshes and wetland and upland forests. Within the constraints of a limited data set, our objective is to advance the understanding of InSAR products to identify change and enhance coastal land cover classification and dynamics.

\section{Study Area}

The analysis was primarily centered on the St. Marks National Wildlife Refuge (NWR) located in the Big Bend coastal region of Florida (Fig. 1). Juncus roemerianus (black needlerush, saline marsh) is the dominant vegetation and accounts for the bulk of biomass in most salt marshes along the northeastern Gulf of Mexico. Inland beyond the black needlerush salt marsh, Cladium jamaicense (sawgrass, fresh marsh), pine-palmetto fringe forest, pine, hardwoods, and scrub shrub dominate, depending on the earlier human activity and local drainage (Ramsey et al., 1998). Black needlerush is characterized by thin, nearly vertical stalks of different densities and heights, depending on marsh maturity and height above mean sea level (Ramsey et al., 1999; Ramsey et al., 2004). Field experience showed the nearly monotypic black needlerush salt marsh transgressed from tall, dense marsh to short, less dense marsh with distance from tidal drainage and nearness to the transitional palmetto and pine forest interface. Sawgrass fresh marsh is a broadleaf plant that forms a mostly vertical canopy; the plant dies in the winter but retains the canopy structure until spring turnover and renewal. In the region, two major forest types occur: (a) wetland forests that include intermittently flooded bottomland hardwoods and a scattering of nearly permanently inundated cypress forests; and (b) upland forests dominated by pines and limited and less extensive stands of upland hardwoods. Structurally, pines have needleleaves and excurrent structures, producing a cone-shaped crown. Pine forests display a fairly to highly nonuniform canopy, with frequent gaps ranging in size from a few to 10 meters. Pine forests remain leaf-on throughout the year, although the 


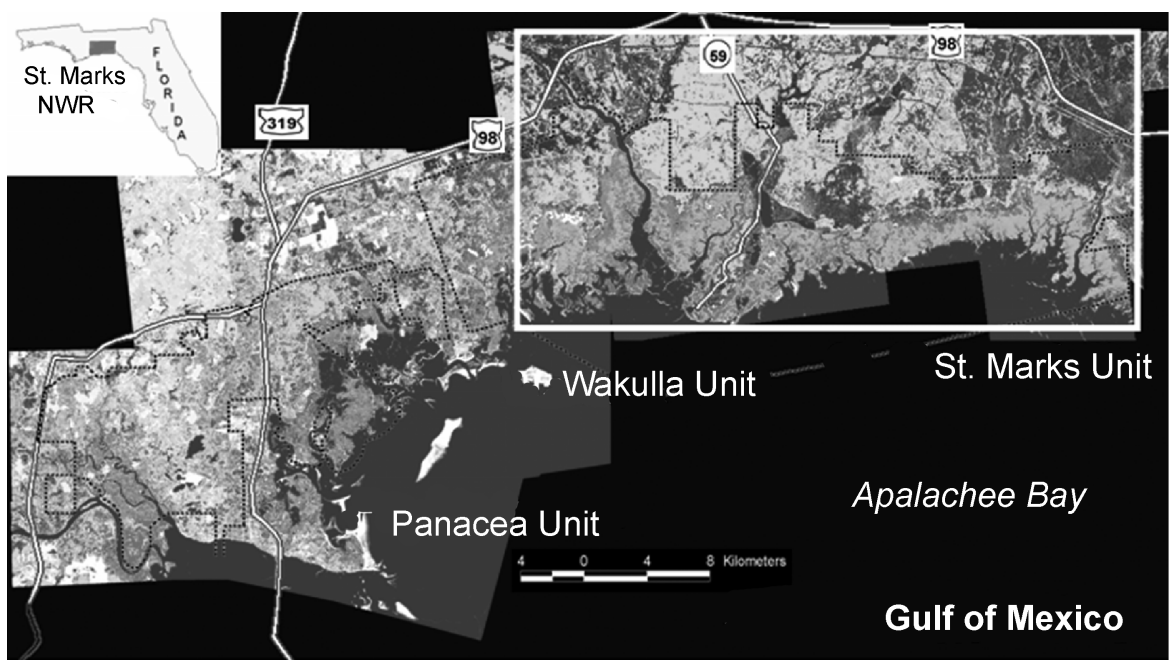

Fig. 1. St. Marks National Wildlife Refuge. The outlined region contained the most image comparisons.

understory and within-gap vegetations, especially fresh marsh grasses, display distinct seasonality. Structurally, bottomland and upland hardwoods have broad leaves and decurrent structures where the lateral branches form a wide and bell-shaped crown. Compared to the pine forests, bottomland and upland hardwoods tend to be taller and exhibit more uniform and denser canopies. In addition, these deciduous hardwood forests lose their leaves in late winter before the spring renewal. A mixed scrub shrub and pine/palmetto forest class often occurs as a transition from fresh marsh to pine forest.

\section{METHODS}

\section{Interferometric Processing}

In 1995, the European Space Agency (ESA) linked the ERS-1 and ERS-2 in a tandem (repeat-pass) mission (C band [5.66 cm], VV polarization, $23^{\circ}$ incidence angle). During the tandem operations, ERS-1 and ERS-2 (ERS-1/2) SAR images were collected 24 hours apart at a 35-day revisit interval, providing the unique opportunity for high-frequency SAR intensity and interferometric data analyses (www.esa.int/esapub/bulletin/bullet83/duc83.htm).

Four of the ERS-1/2 tandem image pairs were obtained for this study (Table 1, Fig. 2). The raw SAR signal data of these pairs were processed (focused) to the Single-Look Complex (SLC) format. The SLC preserves the amplitude and phase of the backscattered signal for each pixel as a complex number. All perpendicular baselines were less than the critical baseline length of $1100 \mathrm{~m}$ (Table 1). In each image pair, one of the two complex InSAR images was registered (aligned and resampled) to the second, and the complex interferogram was constructed by inferring the registered complex images. The phase was unwrapped from the $2 \pi$-repeat cycle. Adaptive filters that respond to the changing land cover were applied to increase the statistical accu- 
RAMSEY ET AL.

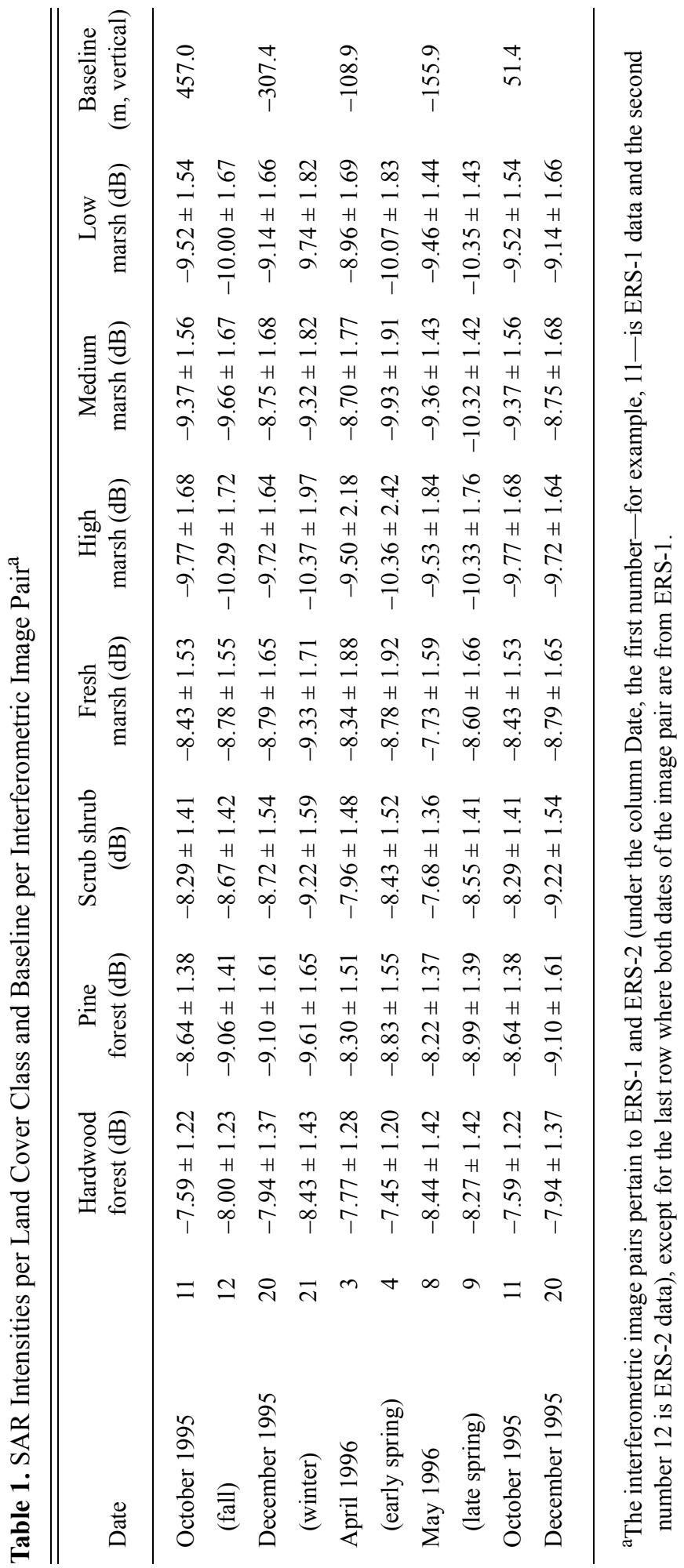




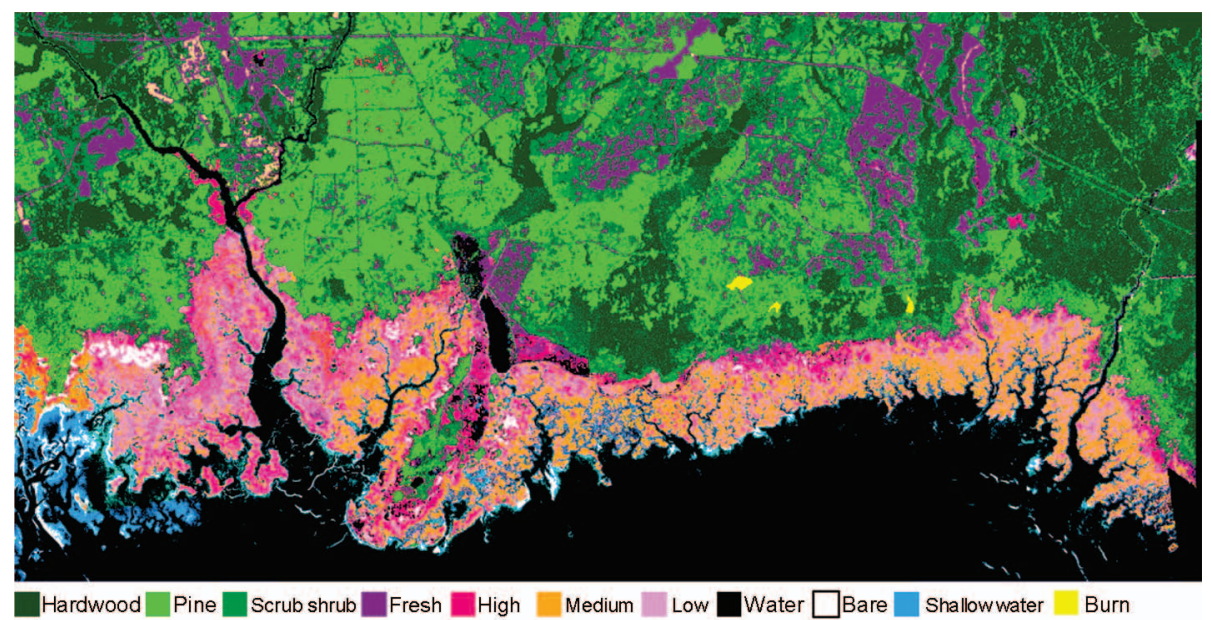

Fig. 2. Classified landscape map of the NWR St. Marks Unit (Ramsey et al., 1998).

racy of the intensity, coherence, and phase estimates, and thereby enhance their association with land cover features (Allen, 1995; Hagberg et al., 1995; Wegmuller and Werner, 1997; Lee et al., 2003). The ERS-1 SAR intensity data were corrected for antennae falloff, calibrated with calibration constants provided by the ESA, and transformed to ground range representation. All interferometric image products were produced at a $40 \mathrm{~m}$ spatial resolution and were rectified to a Universal Transverse Mercator projection common to the classified map. Visual inspection showed subpixel correspondence between the constructed interferometric maps and the classified map.

\section{Land Cover Classification Sources}

Lacking a ground-based accuracy assessment in the west and considering the change in land cover feature dominance from east to west, we limited our comparison with the derived interferometric products to the eastern Wakulla and the entire St. Marks units of the St. Marks NWR (Fig. 2). The classified land cover map was created from a Landsat Thematic Mapper (TM, $25 \mathrm{~m}$ pixel), an ERS-1 SAR (25 m), and color infrared photography $(3.0 \mathrm{~m})$ collected in 1993 and produced at a $10 \mathrm{~m}$ pixel resolution with classification accuracies around $75 \%$ or higher (Ramsey et al., 1998). In comparison to single sensor-based classifications, the image data combination improved classification in the monotypic saline marsh and forest classes. Thirteen classes were identified from forest to marsh to water. Forest classes included hardwoods and pine classes, and the scrub shrub transition class containing a heterogeneous mix of marsh, grasses, and assorted shrubs and pine and palmetto trees of various heights (class referred to as scrub shrub). Marsh classes included sawgrass fresh marsh and black needlerush saline marshes. The saline marsh was divided into high, medium-high, medium, and low classes. Identification of these saline marsh classes was based on field experience and distances from flooding influences (Ramsey, 1995; Ramsey et al. 1998). The sand-flat class included shallow water sand flats, oyster bars, bare fields, and high marsh sand flats. 
In order to improve comparison clarity, water, mixed bare land, and sand flat classes were excluded, and the high medium and medium marsh classes were combined to help diminish the influences of classification error. Even so, classification confusion remained higher in transition classes where spatial coverage was either comparatively small (e.g., fresh marsh in pine forest gaps) or where changes were gradational (e.g., low to medium marsh to high saline marsh). In the latter case, the marsh type did not change, but canopy structure did, as in the transition from medium to low marsh. This classification provided a fair representation of structural changes and a good database for assessing SAR intensity and interferometric classification capabilities.

\section{Application to Water Level Change}

The suite of interferometric images provided a unique opportunity to measure changing flood depths (Lu et al., 2005). Because of the needed juxtaposition of the collection and flooding event and the periodicity of the collections and tidal flooding, limited samples were available during this time period. Concurrent hydrologic measurements at established long-term study sites in the NWR were the original source for calibration; however, water-level measurements were only intermittently available. Hurricane Opal destroyed most of our monitoring equipment right before collection of useable ERS-1/2 images. From our limited hydrology data at the NWR, we know that inland flood waters from Hurricane Opal were still receding up to the October 11, 1995, ERS-1 collection. A similar flooding episode was not indicated during the December ERS-1 SAR image collections. We examined areas outside the NWR that could illustrate how radar interferometry can be used to determine changes in flood depth. In the interferometric image pairs, isolated patches scattered throughout the images exhibited relatively uniform patterns of constant phases. In response to these observations, we collated data relevant to a forest site slightly northeast of the NWR that clearly exhibited phase change. A Landsat TM image collected in May 1995 and a classified National Land Cover Data (NLCD) image (Wardlow and Egbert, 2003) based nominally on a 1992 TM image were acquired. Inspection of the NLCD scene and TM image suggests that the area in question was clear cut between 1992 and 1995 (Figs. 3C and 3D), providing an area conducive for enhancing radar intensity (Ramsey, 1998, 2005).

\section{RESULTS}

Three image pairs were found to be suitable for phase analyses: the October 11 and 12, 1995 image pair (fall), the December 20 and 21, 1995 image pair (winter), and the October 11 and December 20 image pair (fall-winter) (Figs. 4A-4C, Table 1). The interferometric images produced from the ERS-1/2 image pairs collected in 1996 on April 3 and 4 (early spring) and on May 8 and 9 (late spring) exhibited phase aberrations caused by changing atmospheric conditions. Although phase images were unusable, methods were available for producing useful coherence pairs. Following Goldstein and Werner (1998), phase images that represented most of the atmospheric artifacts were produced and subtracted from the original phase images. The resultant 

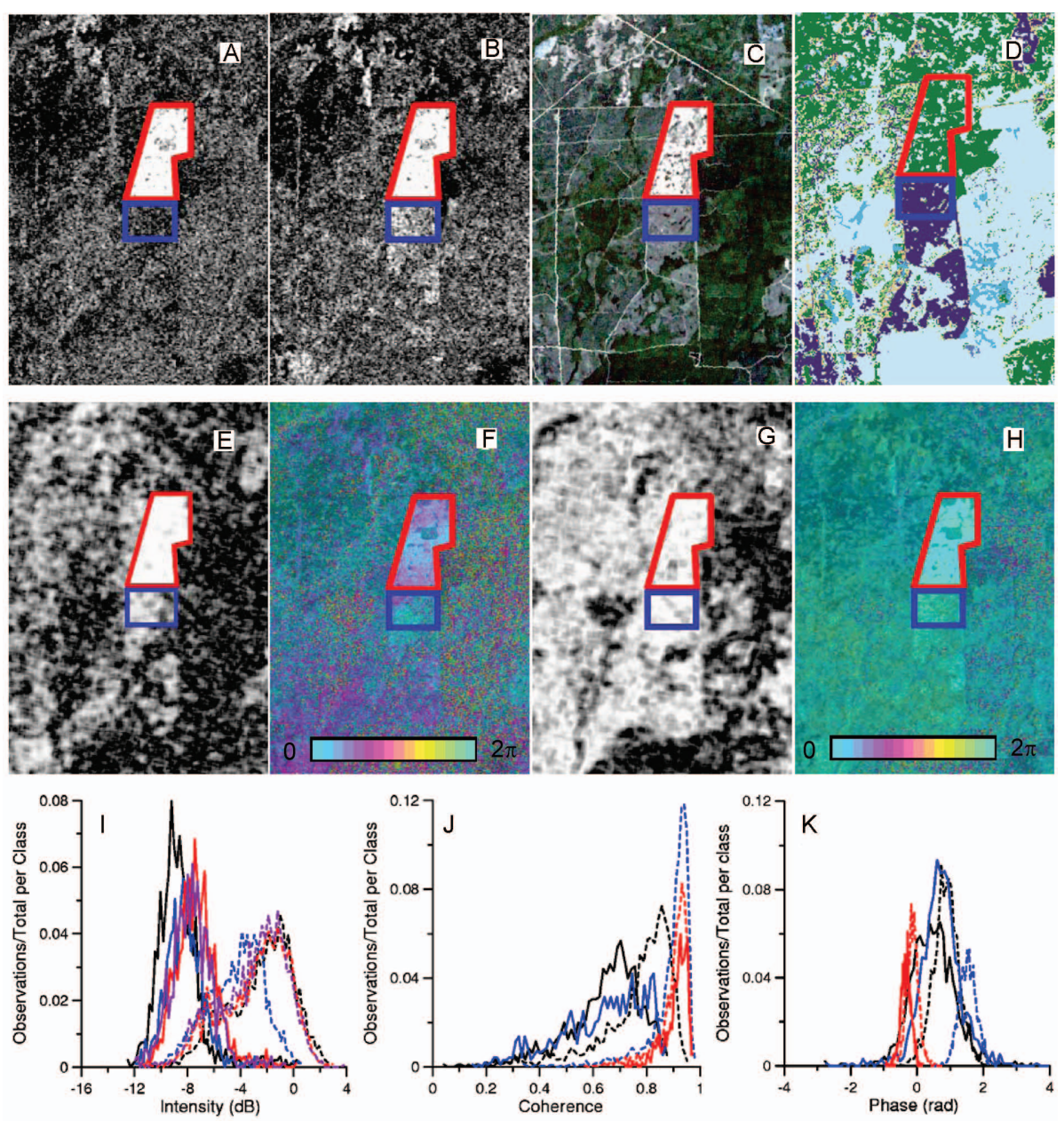

Color legend for Figure 3D (NLCD image): emergent herbaceous wetlands woody wetlands $\square$ evergreen forest mixed forest $\square$ transitional

Fig. 3. Intensity ERS-1 (A) 10/11/1995, (B) 12/20/1995, (C) TM bands 421, and (D) NLCD. Coherence (E) 10/11-12/20 1995, (G) 12/20-12/21 1995, and phase (F) 10/11-12/20 1995 and (H) 12/20-12/21 1995. Intensity (I), coherence (J), and phase (K) histograms of the clear-cut (red image outline, dashed histogram line) and transition class (blue image outline, solid histogram line) areas. The intensity histogram colors are black 10/11/1995, blue 10/12/1995, red 12/20/1995, and purple 12/21/1995. The coherence and phase histogram colors are black 10/11-10/12 1995, blue 10/11-12/20 1995, and red 12/20-12/21 1995. Grey levels are comparable within the intensity images and within the coherence images; brighter denotes higher. North direction is to the top.

phase images were used to produce early and late spring ERS-1/2 coherence images that correctly characterized the coherence and vegetation type association. Intensity images were not expected to be greatly affected by the atmospheric artifacts. 

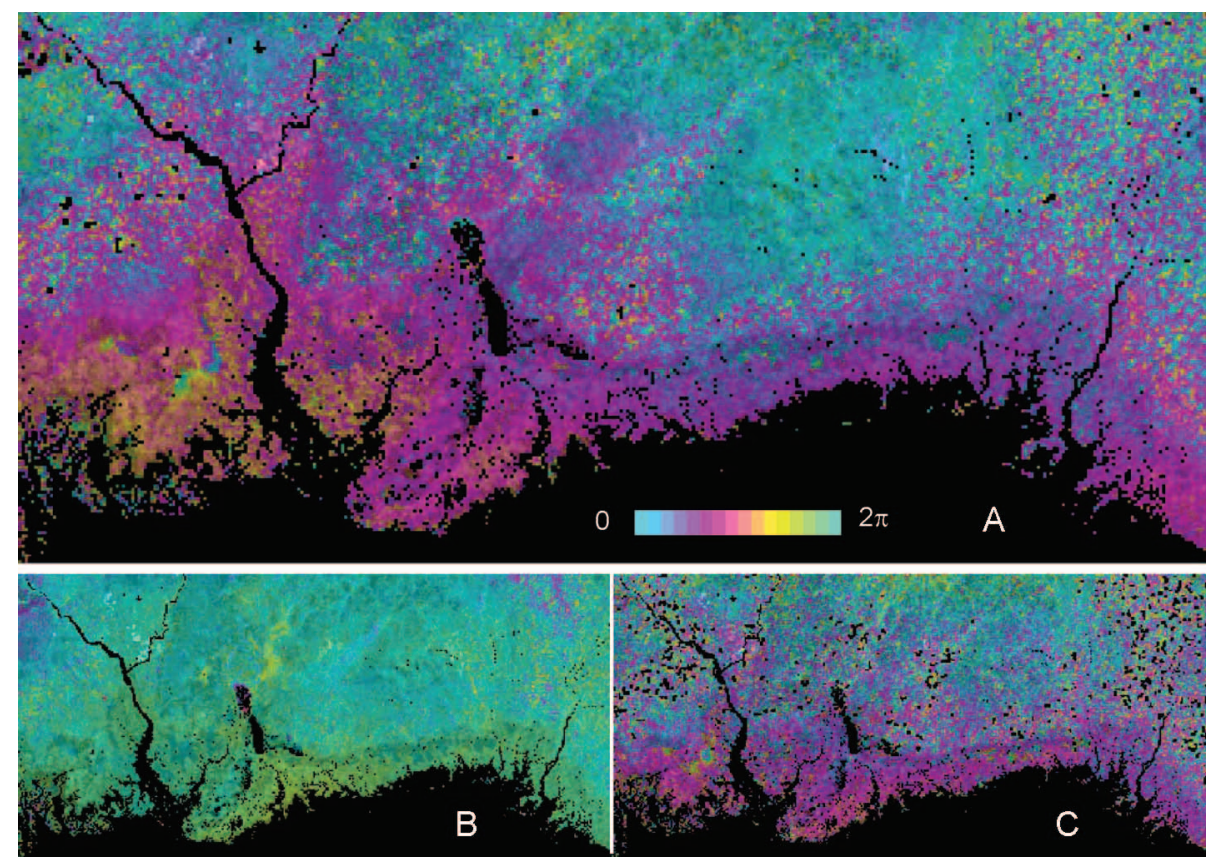

Fig. 4. Interferometric phase (A) 10/11-10/12 1995, (B) 12/20-12/21 1995, and (C) 10/11-12/ 20 1995. A full color phase cycle represents $2 \pi$ radians.

\section{SAR Intensity}

Forest and saline marsh boundaries and hardwood and pine forest stands are easily discernible on the intensity images. Contrast between these cover types is highest in the winter images, moderate in the late fall images, and lowest in the spring images, especially the early spring (Figs. 5A-5D). In most cases, as determined in earlier classifications (Ramsey et al., 1998), hardwoods exhibited the highest-intensity returns, pine and shrub scrub the next highest, and marsh the lowest returns. No saline marsh flooding was identified that was consistent with the documented lowering of the average intensity response from around $-10 \mathrm{~dB}$ to about $-14 \mathrm{~dB}$ (Ramsey, 1995).

Well-formed intensity histograms provided the validation for comparisons based on univariate statistics (Table 1, Figs. 6A and 6B). Outside the late spring hardwood class, ERS-1 mean intensity was lower than the ERS-2 mean by up to $1.2 \mathrm{~dB}$ (Table 1). Differences between means in the winter image pair exhibited the highest consistency across all classes, ranging between about $0.5 \mathrm{~dB}$ and $0.7 \mathrm{~dB}$. Mean differences were slightly lower in the fall, particularly low in the early and late spring hardwood class, and excluding hardwoods, were higher in the later spring and still higher in the early spring saline marsh classes.

Accompanying the relatively lower mean response, except for the spring hardwood class, variance of the mean was higher in the ERS-2 versus ERS-1 intensity image between about $0.1 \mathrm{~dB}$ and $0.3 \mathrm{~dB}$ in the saline classes and around $0.01 \mathrm{~dB}$ to $0.06 \mathrm{~dB}$ in all other classes (Table 1). The highest variance differences were associ- 


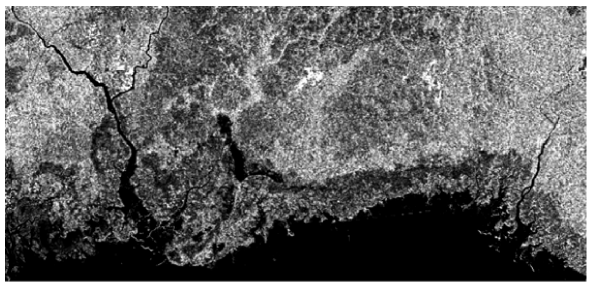

A. ERS-1 SAR intensity 10/11/1995

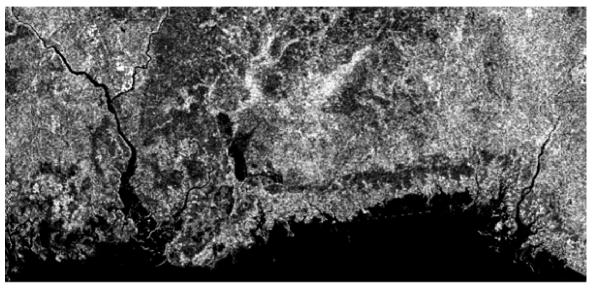

B. ERS-1 SAR intensity 12/20/1995

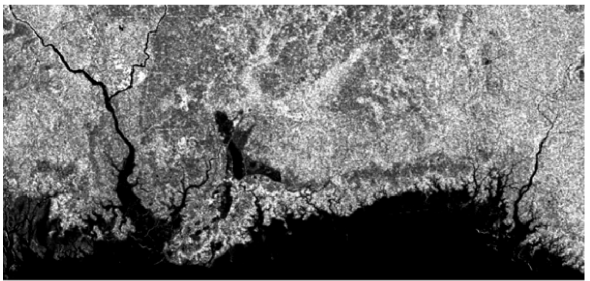

C. ERS-1 SAR intensity 04/03/1996

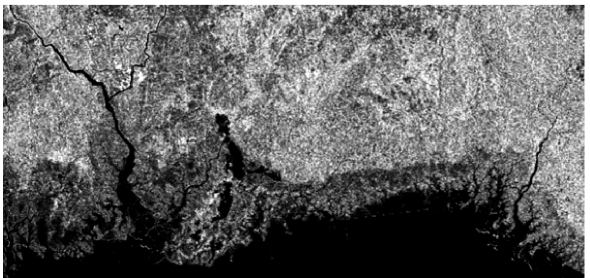

D. ERS-1 SAR intensity 05/08/1996

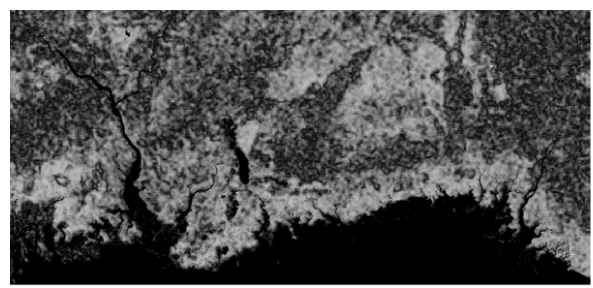

E. Interferometric coherence 10/11-10/12 1995

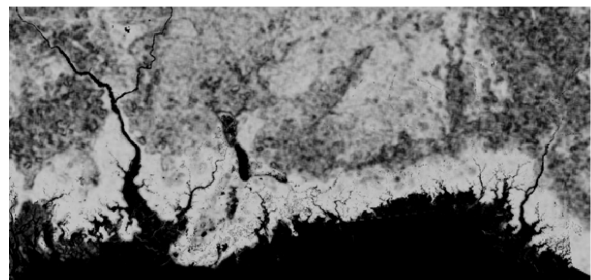

F. Interferometric coherence 12/20-12/21 1995

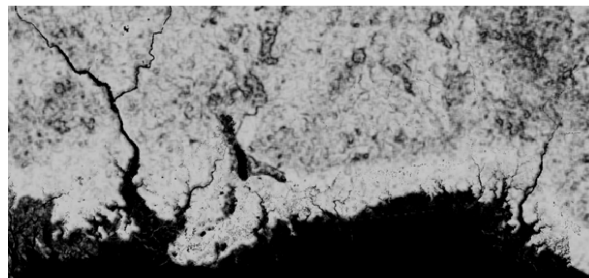

G. Interferometric coherence 04/03-04/04 1996

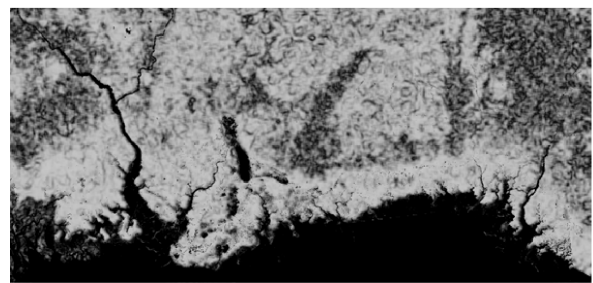

H. Interferometric coherence 05/08-05/09 1996

Fig. 5. Only the first date of all image pairs (ERS-1) intensity images are shown. Grey levels are comparable within the intensity images and within the coherence images; brighter denotes higher. North direction is to the top.

ated with the saline marsh classes in the winter and early spring image pairs, along with an elevated variance difference related to the hardwood class in the spring.

Seasonality associated with ERS-1/2 peak height difference magnitudes was most noticeable in scrub shrubs during the spring, fresh marsh in the fall and winter, and saline marshes most often in winter or early spring (1.0-4.0\% ordinate) (Figs. 6A-6C). In hardwoods and pines, peak differences were lowest in the fall. In addition to peak height, progressive shifts in histogram peak position (abscissa) tracked fall senescence and spring renewal in the fresh marsh, and although less clear, in scrub shrub and pine forests as well (Figs. 7A-7C). The hardwood forest seasonal trend was interrupted by the abnormally lower late spring intensities. Saline marshes showed a more constant peak position response, following trends in optical reflectance (Ramsey et al., 2002; Ramsey et al., 2004); however, mean intensity was slightly elevated 


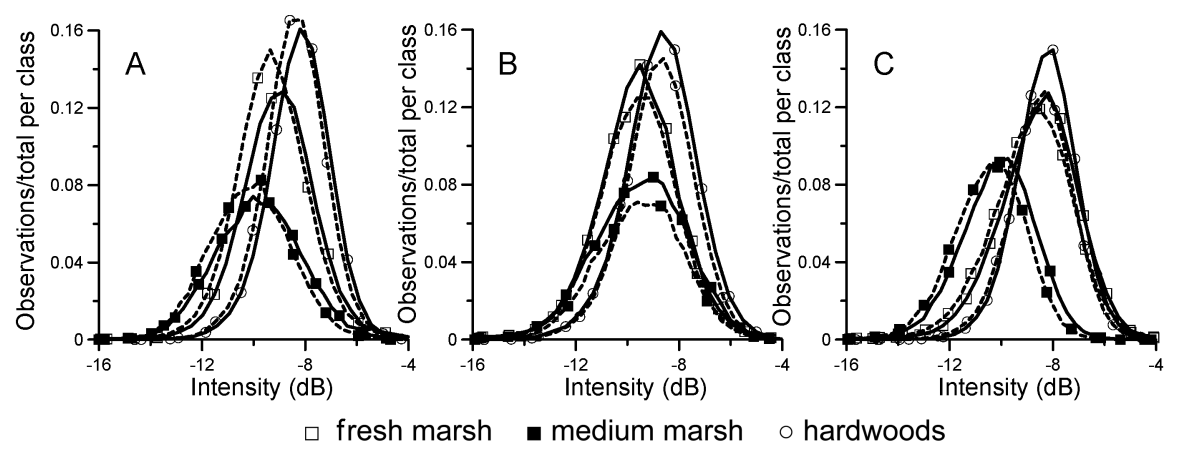

Fig. 6. (A) 10/11-10/12 1995, (B) 12/20-12/21 1995, and (C) 05/08-05/09 1996. Intensity (dB) histogram. The dashed line depicts ERS-1 and the solid line depicts ERS-2 SAR. Histograms were corrected for the ERS-1/2 $0.6 \mathrm{~dB}$ offset.

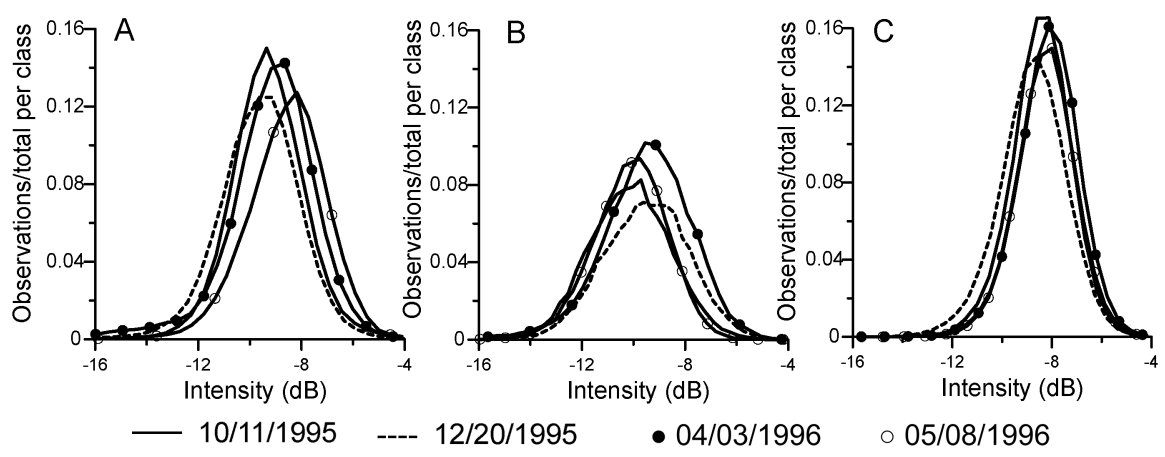

Fig. 7. ERS-1 SAR intensity (dB) histograms for (A) fresh marsh, (B) medium marsh, and (C) hardwoods. Histograms were corrected for ERS-1/2 $0.6 \mathrm{~dB}$ offset.

in the low and medium marsh classes in the winter. Overall, the main seasonal intensity response was shifting peak position in the forest and fresh marsh classes and relatively higher peak intensities in the spring than in fall and winter in the saline marsh classes.

\section{Interferometric Coherence}

As previously discussed, in the coherence comparative analyses, baseline decorrelation influences the results (Table 1). For that reason and for the purposes of this study, outside of some general and qualitative comparisons, coherence was compared per class per image pair.

As in the intensity images, coherence displayed high covariation with land cover features (Figs. 5E-5H). Visibly, forests were associated with lower coherence than marshes. This disparity was best depicted in the fall and in the winter image pairs. The early spring image pair depicted relatively high and the most spatially uniform coherence, while relatively lower coherences were particularly noticeable in the hardwood class in the winter and late spring. Coherence histograms displayed distribu- 
tions that spanned the entire range from those that were sharply defined within a narrow band to those exhibiting broad peaks and wide distributions (Figs. 8A-8E). For all image pairs, saline marshes (especially medium marsh) maintained the highest coherence, and forests (especially hardwoods) maintained the lowest. Fresh and saline marsh coherences were comparable. For the fall-winter interferometric pair with a time separation of 70 days, coherence associated with marsh classes was about 0.4 , while coherence associated with pine and hardwood forests was lower than 0.2 , a threshold indicator for complete decorrelation. Variances on the means were lower in the marsh classes and higher in the forest classes.

\section{Interferometric Phase}

The phase can be used to infer the vegetation height (Slatton et al., 2001). As shown in equations 1 and 2, the sensitivity of phase to the vegetation height is inversely proportional to baseline. Thus, where coherence was maintained, interferograms with higher baselines were used. These conditions were best met in the fall interferometric pair with a $B_{\perp}$ of $457 \mathrm{~m}$ (Table 1, Fig. 4A). In this phase image, $1 \mathrm{~m}$ difference in scatter height (i.e., $\delta h=1 \mathrm{~m}$ ) produced a phase change of about -0.33 radians.

Excluding the fresh marsh class, pine, hardwood, and scrub shrub exhibited similar distributions with a mean of around 0.3 radians, while low, medium, and high marsh classes had means around 2.2 radians (Figs. 9A-9C). These observations indicated that the average scatterer height (phase center) for pine, hardwood, and shrub was about $6 \mathrm{~m}$ higher than that for low, medium, and high marsh classes. Based on field observations, the forest stand heights ranged from around 7.0 to $15.0 \mathrm{~m}$ and saline marsh heights ranged from about 1.0 to $2.0 \mathrm{~m}$. The inferred average difference of $6.0 \mathrm{~m}$ from the phase measurement was from $1.0-8.0 \mathrm{~m}$ less than the field estimates. Surprisingly, the phase distribution of fresh marsh was similar to the forest classes and highly different from the saline marsh classes. No explanation for this similarity was readily apparent.

\section{Hydrology}

Within the forest clear cut, the SAR mean intensity was about $8 \mathrm{~dB}$ higher than that of the transition area (Figs. 3A, 3B, 3I). The clear-cut structure diminished volume decorrelation and promoted the double-bounce mechanism to return the transmitted signal to the sensor, especially when flooded. Accordingly, the coherence over the clear cut was higher than that of the transition area (Figs. 3E, 3G, 3J). This coherence disparity is particularly exemplified in the 70-day interferogram that spanned October 11 and December 20, 1995, where coherence for the clear cut reached 0.95.

The phase images revealed features possibly resulting from surface flooding following Hurricane Opal. The October 11 and December 20, 1995 interferometric pair shows a phase that is about 1.5 radians higher over the clear cut than over the transition area (Figs. $3 \mathrm{~F}, 3 \mathrm{H}, 3 \mathrm{~K}$ ). Because the baseline is about $-51 \mathrm{~m}$, this interferometric pair is not sensitive to changes in scatterer height (i.e., $\delta h=1 \mathrm{~m}$ will result in $\delta \varphi=$ 0.036 radian, equation 2 ). As the change in water level or soil penetration (a function of water content) is vertical, the phase change was related to a water-level decrease of 

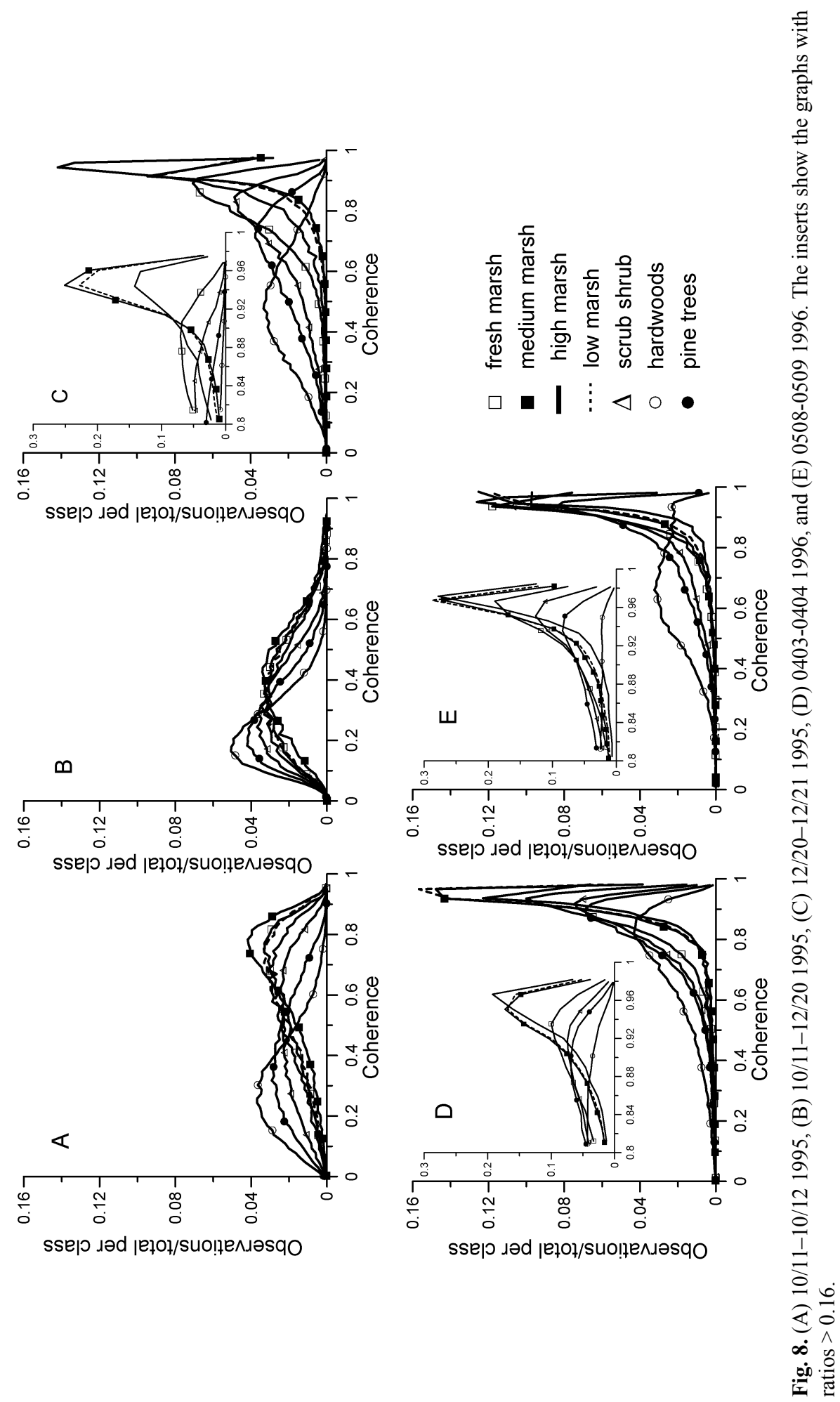


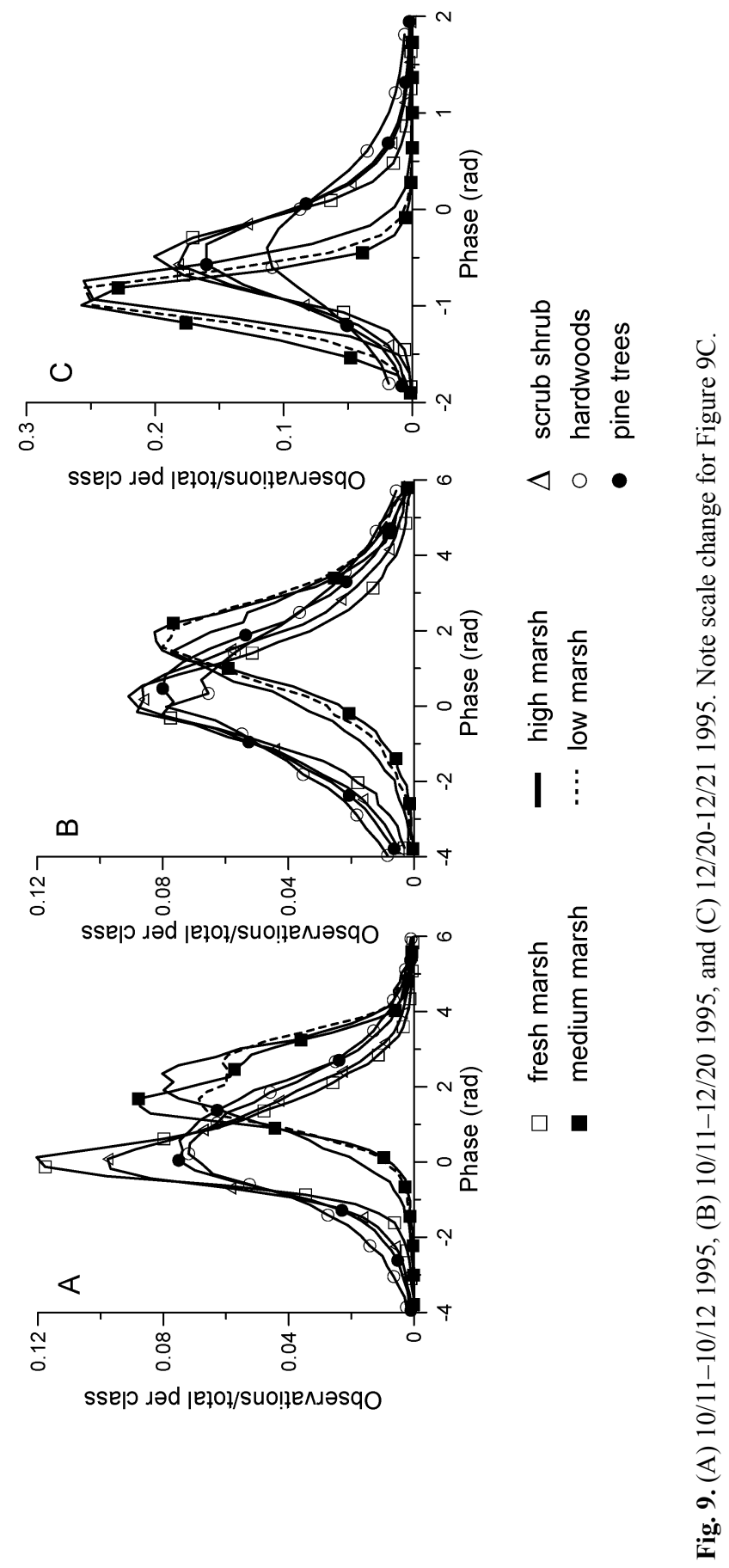


$7.0 \mathrm{~mm}$ or a soil penetration increase that resulted from a decrease in soil moisture content throughout the clear-cut area (i.e., $\delta d=\lambda \delta \varphi / 4 \pi / \cos \theta$ ).

\section{DISCUSSION}

\section{SAR Intensity}

The daily variability and weak seasonal response were two of the most surprising findings among the analyses of the hardwoods and to a lesser extent the pine intensity. Moerately high peak height differences were found between ERS-1/2 image pairs, most often in hardwoods and pines, and more seasonally associated in the scrub shrub and marshes. While hardwood and pine peak height differences were more variable, the intensity variance about the mean was relatively low compared to the marsh classes. Though low, variance increased with peak height, suggesting that increased outliers accompanied the peak increases. These results illustrate the inherent temporal variability in detailed classifications relying on multiple SAR imaging systems. The systematic differences may have resulted from not fully compensating the temporal variations of radar antenna gain in the SLC image due to the lack of an external calibration for the study site. In addition, misalignment of the mean intensities (peak height locations) of the forest and fresh marsh classes increased in the late spring image pair. These shifts may have resulted from incomplete correction of system differences; however, the differences were quite high. More likely, a weather event (e.g., rain, wind) altered the physical state of the canopy leaf layer or caused a change in the volume response.

The seasonal shift in mean intensity was less than expected in the hardwood and fresh marsh classes. In hardwoods, prominent leaf loss may not have occurred until after the December collections, and by early spring the canopy leaf cover may have nearly recovered. The limited collections may not have captured the short transition period of leaf loss and regeneration. Similarly, the dramatic biomass turnover in the fresh marsh may not have occurred concurrently with a collection, but still the spring renewal exhibited a positive intensity shift. In both the hardwood forest and fresh marsh (and somewhat in the pine and scrub shrub) spring biomass addition was reflected as shifts in the intensity peak locations. In the scrub shrub and especially the pine forest classes, however, the spatial juxtaposition of fresh marsh and these forest stands (i.e., mixed pixels) may have caused or at least contributed to the seasonal shifts.

As expected, systematic seasonal intensity shifts associated with the forests and fresh marshes were not observed in the saline marsh classes; however, an unforeseen seasonal increase in peak height was observed. Accompanying the height increase was a higher difference in mean position in the spring ERS-1/2 pairs. As noted, flooding substantially decreases the intensity (Ramsey, 1995), but differences that high were not observed. Residual and scattered pooling of flood waters can dampen the SAR return from these marshes; however, this is most likely when flood frequency is relatively higher (Ramsey, 1995). A sudden change in pooling seems unlikely in one day. Both results point out unexpected seasonal intensity changes, and both seemed to 
imply that a change in the saline marsh canopy structure was linked to the higher peak heights and more variable mean positions within image pairs.

\section{Interferometric Coherence}

Although inverted, coherence displayed many of the same trends as in SAR intensity. As cited in other studies, forests were associated with the lower coherences and marsh classes with the highest. The high coherence in the saline marshes could indicate a predominance of surface scatter (Papathanassiou and Cloude, 2003). In accordance with past studies (Hagberg et al., 1995), our limited leaf-on observations in the study area suggested that hardwood forests, generally having a more uniform and spatially denser canopy than pine forests, would have relatively higher coherences than pine forests. On the contrary, coherences of hardwoods representing more pliable canopy elements (temporal decorrelation) and higher canopy extinction coefficients (volume decorrelation) were lower than the stiffer, more open pine forests. In addition, the highest mean differences between intensity pairs portrayed relatively lower coherences, while small intensity differences were depicted by the higher coherences. Qualitatively, differences in peak heights had no clear connection to coherence, and the longer time period of the fall-winter interferogram produced the lowest coherences in all classes. Relative to a single interferometric image pair, the 70-day time difference is not suitable for mapping forest classes and is marginally adequate for mapping marsh grasses with C-band radar; however, these differential responses associated with multiple baselines and temporal interferometric land cover mapping could provide enhanced discrimination of vegetation types.

Contrary to the winter decrease in forest intensities, winter was marked by some of the highest coherences. Even though the winter baseline was lower than that of the fall, it is important that coherence information remained relatively high during a period when optical information content normally declines in senescencing semiannual vegetation. Coherence distributions also differed from intensity distributions. Coherence distribution shapes and associated peaks were highly varied between classes in contrast to the minor differences associated with the intensity distributions. Coherence sensitivity to temporal and volume decorrelation also provided discriminating information. For instance, the winter and especially late spring coherence images displayed noticeable changes in hardwood coherence. In contrast, only a slight anomaly was depicted in the hardwood mean intensity difference. Further, volume decorrelation indicated easier separation of hardwood from pine forest even during the spring, when intensity contrast was especially low.

\section{Interferometric Phase}

The variability of the phase centers representing returns from all canopy depths is expressed in the phase histograms. In this sense, the shape of the histogram conveys information about the canopy structure within each class. Within a class and without change in canopy height, changing phase centers are connected to changes in the canopy extinction coefficient or the penetration potential of the radar into the canopy depths. Excluding leaf-off periods, normally, hardwoods are associated with higher 
extinctions, and thereby exhibit higher phase centers than do pines that have more open canopies.

We estimated that the phase centers $\sim 6.0 \mathrm{~m}$ associated with the forest classes relative to the saline marsh classes were 1.0-8.0 m lower than the expected average stand heights. These differences resulted from penetration of C-band SAR into the forest canopy lowering the phase centers. As previously discussed, both forest stand height and the associated canopy extinction coefficient variabilities can independently, or in combination, change the resultant phase center. Even though that ambiguity existed, our results suggest broader phase distributions associated with forests and narrower distributions associated with marshes, especially the saline marshes. Coherence increases from marsh to forest classes, so the phase comparison is not unambiguous. Resolution of the canopy height and structure ambiguities would require the multiple baseline image data to be combined with ground-based measurements or adjacent targets of a well-estimated phase. If the ambiguity were removed, and excluding the forest and marsh canopy depth differences, then the broader forest distributions would indicate higher variability in forest than marsh canopy structures, and the different phase centers would indicate height differences. Without appropriate calibration data, correspondence of intensity, coherence, and phase can only be relatively compared within the forest and fresh marsh and within the saline classes.

\section{Improving Land Cover Classifications}

We have documented and provided numerous distinct and general associations of the coastal land cover features and SAR intensity as well as coherence and phase for comparison (Figs. 2-5, 8-9). First, intensities were higher in the forests than the marshes, and highest in the hardwoods. Although a fairly common result, we focus on the contrast between land covers provided by SAR intensity versus coherence and phase. A higher contrast improves land cover separation and thereby classification accuracies, and, as discussed, both coherence and phase outperform intensity in denser canopies. Second, although coherence and phase interpretations vary with baseline, the selective association of coherence and phase magnitudes and spatial texture indicates an ability to use these measures for land cover classification. For instance, only marshes retained moderate coherence $(>0.4)$ in the fall-winter image pair, all but hardwoods maintained high coherence $(>0.7)$ in the winter image pair. Third, outside the unique ability to provide canopy height information, the spatial texture of phase and coherence provides canopy structure data via the extinction coefficient, and phase may outperform coherence in taller canopies. For example, phase image depictions and histogram results indicated that forest canopy structures were less spatially uniform than marshes and only hardwoods exhibited highly textured coherence and phase patterns in the winter. We applied these capabilies and results to a small section of the coastal land cover in order to illustrate how interferometric products may provide additional information relative to optical land cover mapping (outside of collection constraints) and what possible advantages coherence and phase (neglecting vegetation height and deformation) offer with respect to SAR intensity, even when high-frequency SAR is available. To help illustrate some differences in the optical, SAR, and interferometric products, we used the area centered on the forest clear cut (Fig. 3). The intensity, coherence, and phase distributions indicate that the 
transition class (as designated in the NLCD metadata file) was dominantly marsh or an older re-growth containing marsh.

Intensity distributions showed higher returns from the clear cut than from the transition class area (Figs. 3A, 3B, 3I); indicating an enhanced return, supporting a double-bounce return mechanism. In addition, abnormal decreases in the fall transition class ERS-1 (10/11/1995) and fall clear cut ERS-2 (10/12/1995) intensities were depicted (Fig. 3I). Rainfall and the accompanying canopy wetness can cause an intensity decrease (Hobbs et al., 1998); however, in this case, attenuated returns should have occurred in both the clear cut and transition areas. Given the documented flood attenuation of backscatter return in these marshes (Ramsey, 1995), the rather large decrease suggests sub-canopy flooding or extensive pooling in the transition area. The next day decrease in the clear-cut intensity implies a dampened double-bounce return. Although the double-bounce mechanism occurs without flooding, flood abatement could have attenuated the clear cut responses. An unlikely alternate explanation would require an abrupt decrease in soil moisture content in one day. A more likely explanation is that flooding or pooling abated between the fall ERS-1/2 collections.

Further support for the flood abatement is found when considering our coastal hydrology measurements that showed continued flood recession during the fall ERS1/2 collections, abnormally lower ERS-1 versus ERS-2 marsh intensity returns (Fig. $6 \mathrm{~A}$ ), and finally, relatively lower returns in the northwest of the clear-cut and transition subset image area (Figs. 3A and 3B). In the NLCD-1992, the northwest corner was classified as evergreen forest (Fig. 3D); however, the 1995 Landsat TM image (Fig. 3C) did not indicate a forest area. Similar to the transition area, intensity, coherence, and phase indicated that the northwest area was dominated by marsh with scattered forest stands (Figs. 3E-3H). Although both the northwest area and the transition area exhibited flooding or remnant pooling in the fall ERS-1 intensity image, a higher marsh canopy extinction or higher water elevation could have caused the relative intensity difference. Visibly, this high attenuated extent decreased in the fall ERS-2 image (not shown), indicating flood recession. Flooding in the northwest area was also indicated in the winter intensity image, although not in the transition area (Fig. 3B). Intensity histograms support the lack of extensive winter flooding in the transition area and indicated enhanced winter returns in the forest clear cut relative to the fall ERS-2 intensity image. As previously discussed, even though not ideal for determining changes in water level, the fall-winter phase indicated a minimal decrease in flood depth or soil moisture change from the fall to winter collections. Contrary to the fall intensity images, no corroborative evidence was available to support clear-cut flooding during the winter collections. In more open clear-cut areas, saturated soil conditions can promote double-bounce returns mimicking flood double-bounce enhancements. As noted in the intensity images, flooding still existed in the northwest during the winter collections, suggesting that even if flooding was not present in the clear-cut area, saturated soil conditions were quite likely.

Outside the clear-cut and transition areas, the most ubiquitous NLCD class was wetland forest, followed by evergreen, and then mixed forest (Fig. 3D). In comparisons to the classified map, we showed that only fresh and saline marsh classes retained moderately high coherences in the fall-winter coherence image (Fig. 8B). By analogy, the higher coherence areas depict marsh on these images; the remaining areas indicate forest classes (Fig. 3E). This spatial distribution of high coherences 
supported the interpretation of the lower intensities in the northwest being related to flooded marsh. In addition, hardwoods exhibited the lowest coherences in the winter coherence image, while all other classes were represented by higher coherences (Fig. $8 \mathrm{C})$. The darkest forest areas on the winter coherence image are almost certainly dominated or have a high stand composition of hardwoods (wetland forest) (Fig. 3G). Taken together, the forest distribution determined from the fall-winter coherence that followed the NLCD woody wetland class distribution and the winter coherence image highlighting hardwood forests indicated the NLCD wetland forest class also included many evergreen forest (pine) and mixed forest classes. In addition, the NLCD evergreen and mixed forest classes seemingly incorporated older re-growths (scrub shrub) or marshes. The phase texture distribution is consistent with these land cover distribution interpretations.

\section{CONCLUSIONS}

The nature and uniqueness of interferometric products in identifying land cover type and biophysical variability were illustrated in a comparison to a detailed optical classification. An assessment of daily and seasonal changes provided a unique examination of SAR intensity stability as related to vegetation type (class). Coherence stabilities, distributions, and trends indicated the value of coherence for land cover classifications and provided examples of its role in assessing the confidence of phase information. Phase provided a unique perspective of the land cover through its relationship to canopy extinction and height, as well as through its response to changes in water levels or soil moisture content given special circumstances.

Visual inspection showed close correspondence between the major cover types and the intensity, coherence, and phase images. Overall, forest and saline marsh boundaries and hardwood and pine forest stands were discernible, though more easily in the fall and winter than in the spring. Forests, particularly hardwoods, exhibited the highest intensities and marshes exhibited the lowest, especially the saline marshes. Small but progressive shifts in intensity tracked fall senescence and spring renewal, most notably in the fresh marsh, and then the scrub shrub, pine forest, and hardwood forest classes. Saline marshes exhibited a fairly constant response in intensity; however, intensity distributions became more uniform in the spring than in the fall and winter. Intensity distribution variability per class and between ERS-1/2 image pairs suggested an inherent temporal variability that could impact detailed classifications relying on multiple SAR imaging systems, even closely aligned systems.

Coherence was inverted with respect to intensity. Forests displayed lower coherences, and marsh classes displayed the highest. On average, coherences within the hardwood class were lower than pine forests. In the fall-winter pair with a 70-day separation, only the scrub shrub and marsh classes maintained a functional coherence (about 0.4 ). In the late spring, an abrupt decrease of coherence was observed primarily in the hardwood class, containing the most pliable canopy elements. This decrease was revealed only as a very slight decrease in the intensity distributions. This enhanced sensitivity of coherence versus intensity was further illustrated in the winter collections when intensity contrast was low while coherence contrast between land cover types remained fairly high. Coherence distributions varied highly between classes in contrast to the minor differences associated with the intensity distributions. 
Temporal and volume decorrelations of the coherence provided land cover discrimination, particularly when intensity contrast was low, as in the spring images.

Consistent phase information largely responded to the level of coherence within each class and revealed canopy structure parameters related to different classes and within each class. The phase center for hardwood and pine was about $6 \mathrm{~m}$ higher than it was for the saline marsh classes. This difference was lower than observed heights and points to SAR penetration within the forest stands. Independent extinction coefficient estimates would provide prediction of canopy heights. As in coherence distributions, phase distributions portrayed a high sensitivity to within-class variance that would be useful in canopy structure and biophysical mapping.

Intensity, coherence, and phase information were applied to determining the change in water level in a recently clear cut area where flooding and flood abatement were suspected. Abnormal intensity decreases in the marsh and a day later in the forest clear cut indicated flood abatement occurred as documented in coastal hydrologic measurements and as observed in dampened intensity returns in the coastal marsh. Additionally, within the region of high coherence and between the fall and winter collections, the phase indicated a relative water surface height decrease of around $7 \mathrm{~mm}$, or alternatively, a differential change in soil moisture.

In the same clear cut region, we tested the relationships found between our land cover classes and interferometric products on a small area exhibiting recent land cover changes. Changes that were observed on the concurrent Landsat TM image but not on the national classification were reflected on the SAR intensity images. Outside of suspected flooded areas, the highest intensity contrast in fall and winter, reflecting land cover changes, was most often $<1 \mathrm{~dB}$ and even less on the low-contrast spring intensity image. In contrast, high changes in coherence depicted the land cover variations. In addition, the differential coherence response per land cover in each image pair allowed the determination of marsh, scrub shrub, bottomland hardwood, and pine forest classes that were not clearly discriminated in the intensity images. Gradational coherence changes within these classes indicated that higher detailed discrimination was possible; however, only broad class discrimination was examined. Changes in phase further corroborated the class predictions and could potentially add class structure information. These class predictions were not directly validated in the clear-cut subset area. However, the noted changes in intensity, coherence, and phase uncovered in our comparison with a validated vegetation map were unique and compelling evidence of the predicted associations.

The addition of coherence and phase to SAR intensity and comparisons to a validated and spatially detailed coastal map provided new information on radar interferometry. Such information may be used to extend and advance the capabilities of optical sensors in mapping coastal resources and coastal dynamics by expanding the set of useful information available from SAR satellite systems. The SAR systems offer 24-hour-a-day collections nearly independent of the weather conditions that typically severely hamper the reliance on optical systems for detailed and consistent coastal resource management. The temporal advantage of SAR systems is enormous. With increasing SAR and InSAR system capabilities, SAR and InSAR resource mapping are moving from being the collateral to being the primary data source for mapping coastal regions. Results of this study show that further extension of current 
SAR and InSAR capabilities and addition of new, multiple-feature SAR systems will increasingly improve mapping detail, accuracy, and functionality.

\section{ACKNOWLEDGMENTS}

We thank Frank Baarnes, Madlena Hakobyan, and Sijan Sapkota (IAP World Services, Inc.) and Oh-ig Kwoun (SAIC) for contributing to the completion of this work. We greatly appreciate and especially thank Anthony Lewis, Bhaskar Ramachandran, and Eugene Fornigh for their concise and thoughtful reviews. We also thank U.S. Geological Survey personnel Beth Vairin and IAP personnel Victoria Jenkins and Jarita Davis for editing the manuscript. Partial funding for this study was granted through the U.S. Geological Survey's Venture Capital Funds Program and Land Remote Sensing Program.

\section{REFERENCES}

Allen, C., 1995, "Interferometric Synthetic Aperture Radar," IEEE Geosciences and Remote Sensing Society Newletter, 96:6-13.

Armour, B., Tanaka, A., Ohkura, H., and G. Saito, "Radar Interferometry for Environmental Change Detection," in Remote Sensing Change Detection: Environmental Monitoring Methods and Applications, Lunetta, R. and C. Elvidge, (Eds.), Ann Arbor, MI: Ann Arbor Press, Inc., 245-279.

Baran, I., 2004, Advanced Satellite Radar Interferometry for Small-Scale Surface Deformation Detection, Doctoral thesis, Western Australia School of Mines, Department of Spatial Sciences, Curtin University of Technology, Bentley, Australia, $178 \mathrm{p}$.

Cloude, S. and K. Papathanassiou, 1998, "Polarimetric SAR Interferometry," IEEE Transactions on Geoscience and Remote Sensing, 36(5):1551-1565.

Dobson, C., Ulaby, F., and L. Pierce, 1995, "Land-Cover Classification and Estimation of Terrain Attributes Using Synthetic Aperture Radar," Remote Sensing of Environment, 51:199-214.

Elachi, C. T., 1988, Spaceborne Radar Remote Sensing: Applications and Techniques, New York: IEEE Press.

Goldstein, R. M. and C. L. Werner, 1998, "Radar Interferogram Filtering for Geophysical Applications," Geophysical Research Letters, 25(21):4035-4038.

Hagberg, J., Ulander, L., and J. Askne, 1995, "Repeat-Pass SAR Interferometry over Forested Terrain," IEEE Transactions on Geoscience and Remote Sensing, 33(2):331-340.

Hajnsek, I., Papathanassiou, K., and S. Cloude, 2003, “A Hybrid Scattering Model for Surface Parameter Estimation using Polarimetric SAR Interferometry," in Proceedings, Geoscience and Remote Sensing Symposium, IGARSS ’03, 2:693695.

Hobbs, S., Ang, W., and C. Seynat, 1998, "Wind and Rain Effects on SAR Backscatter from Crops," in ESA SP-441 Workshop on the Retrieval of Bio- and Geophysical Parameters, Noordwijk, The Netherlands: ESTEC, 185-189. 
Hoen, E. and H. Zebker, 2000, "Penetration Depths Inferred from Interferometric Volume Decorrelation Observed over the Greenland Ice Sheet," IEEE Transactions on Geoscience and Remote Sensing, 38(6):2571-2582.

Jensen, J., Ramsey, E., Mackey, H., Christensen, E., and R. Sharitz, 1987, "Inland Wetland Change Detection using Aircraft MSS data," Photogrammetric Engineering and Remote Sensing, 53:521-528.

Jensen, J., 2000, Remote Sensing of the Environment. An Earth Resource Perspective, Englewood Cliffs, NJ: Prentice Hall.

Kasischke, E. and L. Bourgeau-Chavez, 1997, "Monitoring South Florida Wetlands Using ERS-1 SAR Imagery," Photogrammetric Engineering and Remote Sensing, 63(3):281-291.

Klemas, V., Dobson, J., Ferguson, R., and K. Haddad, 1993, “A Coastal Land Cover Classification System for the NOAA Coastwatch Change Analysis Project," Journal of Coastal Research, 9(3):862-872.

Lee, J., Cloude, S., Papathanassiou, K., Grunes, M., and I. Woodhouse, 2003, "Speckle Filtering and Coherence Estimation of Polarimetric SAR Interferometry Data for Forest Applications," IEEE Transactions on Geoscience and Remote Sensing, 41(10):2254-2263.

Lewis, A., Henderson, F., and D. Holcomb, 1998, "Radar Fundamentals: The Geoscience Perspective," in Principles and Applications of Imaging Radar, Henderson, F. and A. Lewis (Eds.), New York, NY: John Wiley \& Sons, Inc. (Manual of Remote Sensing, 3:131-181).

Liu, J., Mason, P., Hilton, F., and H. Lee, 2004, "Detection of Rapid Erosion in SE Spain: A GIS Approach Based on ERS SAR Coherence Imagery," Photogrammetric Engineering and Remote Sensing, 70(10):1179-1185.

Lu, Z., Crane, M., Kwoun, O., Wells, C., Swarzenski, C., and R. Rykhus, 2005, "Cband radar observes water-level change in swamp forests," EOS (Transactions of the American Geophysical Union), 86(14):141-144.

Lu, Z. and J. Freymueller, 1998, "Synthetic Aperture Radar Interferometry Coherence Analysis over Katmai Volcano Group, Alaska," Journal of Geophysical Research, 102(B12):29,887-29,894.

Lunetta, R., Lyon, J., Guindon, B., and C. Elvidge, 1998, "North American Landscape Characterization Dataset Development and Data Fusion Issues," Photogrammetric Engineering and Remote Sensing, 64(8):821-829.

Lyon, J. and J. McCarthy, 1981, "Seasat Imagery for detection of coastal wetlands," in Proceedings of the Fifteenth International Symposium on Remote Sensing of Environment, Ann Arbor, MI: ERIM, 1475-1485.

Massonnet, D. and K. Feigl, 1998, "Radar Interferometry and its Application to Changes in the Earth's Surface," Review of Geophysics, 36(4):441-500.

Moghaddam, M. and K. McDonald, 2003, "Mapping Wetlands of North American Boreal Zone from Satellite Radar Imagery," in Proceedings, Geoscience and Remote Sensing Symposium, IGARSSS '03, 261-263.

Papathanassiou, K. and S. Cloude, 2003, "The Effect of Temporal Decorrelation on the Inversion of Forest Parameters from Pol-InSAR Data," in Proceedings, Geoscience and Remote Sensing Symposium, IGARSS '03, 3:1429-1431. 
Ramsey, E., III, 1995, "Monitoring Flooding in Coastal Wetlands by Using Radar Imagery and Ground-Based Measurements," International Journal of Remote Sensing, 16:2495-2502.

Ramsey, E., III, 1998, "Radar Remote Sensing of Wetlands," in Remote Sensing Change Detection: Environmental Monitoring Methods and Applications, Lunetta, R., and C. Elvidge (Eds.), Ann Arbor, MI: Ann Arbor Press, Inc., 211243.

Ramsey, E., III, 2005, "Remote Sensing of Coastal Environments," in Encyclopedia of Coastal Science, Encyclopedia of Earth Sciences Series, M. L. Schwartz (Ed.), Dordrechlt, The Netherlands: Kluwer Academic Publishers, 797-803.

Ramsey, E., III, Hodgson, M., Sapkota, S., and G. Nelson, 2001a, "Forest Impact Estimated with NOAA AVHRR and Landsat TM Data Related to an Empirical Hurricane Wind Field Distribution," Remote Sensing of Environment, 77(3):279292.

Ramsey, E., III and S. Laine, 1997, "Comparison of Landsat Thematic Mapper and High Resolution Photography to Identify Change in Complex Coastal Marshes," Journal of Coastal Research, 13(2):281-292.

Ramsey, E., III, Nelson, G., Baarnes, F., and R. Spell, 2004, "Light Attenuation Profiling as an Indicator of Structural Changes in Coastal Marshes," in Remote Sensing and GIS Accuracy Assessment, Lunetta, R. and J. Lyon (Eds.), New York, NY: CRC Press, 59-73.

Ramsey, E., III, Nelson, G., and S. Sapkota, 1998, "Classifying Coastal Resources by Integrating Optical and Radar Imagery and Color Infrared Photography," Mangroves and Salt Marshes, 2(2):109-119.

Ramsey, E., III, Nelson, G., and S. Sapkota, 2001b, "Coastal Change Analysis Program Implemented in Louisiana," Journal of Coastal Research, 17(1):55-71.

Ramsey, E., III, Nelson, G., Sapkota, S., Laine, S., Verdi, J., and S. Krasznay, 1999, "Using Multiple Polarization L Band Radar to Monitor Marsh Burn Recovery," IEEE Transactions on Geoscience and Remote Sensing, 37(1):635-639.

Ramsey, E., III, Rangoonwala, A., Nelson, G., and R. Ehrlich, 2005, "Mapping the Invasive Species, Chinese Tallow with EO1 Satellite Hyperion Hyperspectral Image Data and Relating Tallow Percent Occurrences to a Classified Landsat Thematic Mapper Land Cover Map," International Journal of Remote Sensing, 26(8):1637-1657.

Ramsey, E., III, Sapkota, S., Barnes, F., and Nelson, G., 2002, “ Monitoring the Recovery of Juncus roemerianus Marsh Burns with the Normalized Vegetation Index and Landsat Thematic Mapper Data," Wetlands Ecology and Management, 10(1):85-96.

Reigber, A., Moreira, A., and K. Papathanassiou, 1999, "First Demonstation of Airborne SAR Tomography using Multibaseline L-band Data," in Proceedings, Geoscience and Remote Sensing Symposium, IGARSS '99, 1:44-46.

Slatton, K., Crawford, M., and B. Evans, 2001, "Fusing Interferometric Radar and Laser Altimeter Data to Estimate Topography and Vegetation," IEEE Transaction s of Geoscience and Remote Sensing, 39(11):2470-2482.

Treuhaft, R., Asner, G., Law, B., and S. Tuyl, 2002, "Forest Leaf Area Density Profiles from the Quantitative Fusion of Radar and Hyperspectral Data," Journal of Geophysical Research, 107(D21):1-13. 
Treuhaft, R., Law, B., and G. Asner, 2004, "Forest Attributes from Radar Interferometric Structure and its Fusion with Optical Remote Sensing," BioScience, 54(6):561-571.

Ulaby, F. and M. Dobson, 1989, Handbook of Radar Scattering Statistics for Terrain, Norwood, UK: Artech House.

Wardlow, B. and S. Egbert, 2003, "A State-Level Comparative Analysis of the GAP and NLCD Land-Cover Data Sets," Photogrammetric Engineering and Remote Sensing, 69(12):1387-1397.

Waring, R., Way, J., Hunt, E., Jr., Morrissey, L., Ranson, K., Weishampel, J., Oren, R., and Franklin, S., 1995, "Imaging Radar for Ecosystem Studies," BioScience, 45(10):715-723.

Wegmuller, U. and C. Werner, 1997, "Retrieval of Vegetation Parameters with SAR Interferometry," IEEE Transactions on Geoscience and Remote Sensing, 35(1):18-24.

Zebker, A. and J. Villasenor, 1992, "Decorrelation in Interferometric Radar Echoes," IEEE Transactions on Geoscience and Remote Sensing, 30(5):950-959. 